University of Minnesota Morris Digital Well

University of Minnesota Morris Digital Well

2019

\title{
The Explicit Formula and a Motivic Splitting
}

David P. Roberts

University of Minnesota - Morris, roberts@morris.umn.edu

Follow this and additional works at: https://digitalcommons.morris.umn.edu/mathematics

Part of the Algebra Commons

\section{Recommended Citation}

David P. Roberts. The Explicit Formula and a Motivic Splitting. >2017 MATRIX Annals (2019), 429-433.

This Article is brought to you for free and open access by the Faculty and Staff Scholarship at University of Minnesota Morris Digital Well. It has been accepted for inclusion in Mathematics Publications by an authorized administrator of University of Minnesota Morris Digital Well. For more information, please contact skulann@morris.umn.edu. 


\title{
The explicit formula and a motivic splitting
}

\author{
David P. Roberts
}

\begin{abstract}
We apply the Guinand-Weil-Mestre explicit formula to resolve two questions about how a certain hypergeometric motive splits into two irreducible motives.
\end{abstract}

\section{Introduction}

The classical explicit formula of Guinand and Weil was generalized to a broader context by Mestre in [1]. This formula applies to any $L$-function satisfying standard analytic properties, and gives a family of formulas for its conductor $N$. Mestre used it to get lower bounds on conductors of abelian varieties. This extended abstract gives an example of how it can be used in more exotic motivic contexts.

The example we pursue here has the form $M=M_{8} \oplus M_{6}$, the factor motives being indexed by their degree. We assume that the associated $L$-functions really do have the required analytic properties, and work numerically to a precision that is adequate for being very confident in the assertions. Presently, we can compute directly with $M$, but not with the individual factors. We know that its conductor is $\operatorname{cond}(M)=2^{15}$ and its local $L$-factor at 2 is just 1 . These numerics imply that one of $M_{6}$ and $M_{8}$ is tame at 2, and the other is minimally wild. Also we know the order of central vanishing is $\operatorname{rank}(M)=2$. This raises two questions:

Q1: $\quad\left(\operatorname{rank}\left(M_{6}\right), \operatorname{rank}\left(M_{8}\right)\right)$ can only be $(2,0),(1,1)$, or $(0,2)$. Which is correct?

Q2: $\left(\operatorname{cond}\left(M_{6}\right)\right.$, cond $\left.\left(M_{8}\right)\right)$ can only be $\left(2^{6}, 2^{9}\right)$ or $\left(2^{7}, 2^{8}\right)$. Which is correct?

The answers are given in the table at the end of this extended abstract. We provide enough computational details so that the reader can both reproduce our answers and attempt analogous calculations for other split motives.

David P. Roberts

University of Minnesota Morris, USA, e-mail: roberts@morris.umn.edu 
2 The motive $M=M_{6} \oplus M_{8}$

One of the points of the talk was to illustrate how the Magma hypergeometric motives package by Mark Watkins lets one compute with hypergeometric motives of large degree. We use Magma language here as well [2], and the reader can repeat most computations using the free online Magma calculator.

To obtain the motive $M$ and its $L$-function $L$, type

$$
\begin{aligned}
& \mathrm{M}:=\text { HypergeometricData ( } \\
& \qquad[1 / 2: \text { i in }[1 \ldots 16]],[0: \text { i in }[1.16]]) ; \\
& \mathrm{L}:=\text { LSeries (M, } 1 \text { : Precision:=10, BadPrimes: }=[<2,15,1>]) ;
\end{aligned}
$$

Here Magma correctly understands that $M$ has good reduction outside of 2 . The optional argument ensures that it has the correct data at 2 as well, that being conductor $2^{15}$ and local $L$-factor 1 . Other possibilities failing badly, correctness of the choice $\langle 2,15,1\rangle$ is confirmed by CheckFunctionalEquation (L) returning 0.0000000000 . The command HodgeStructure ( $\mathrm{L}: \mathrm{PHV})$ says that $M$ has weight $w=15$ with Hodge vector

$$
\left(h^{0,15}, h^{1,14}, \ldots, h^{14,1}, h^{15,0}\right)=(1,1,1,1,1,1,1,0,0,1,1,1,1,1,1,1) .
$$

In particular $M$ can only appear in the cohomology of varieties of dimension $\geq 15$.

In general, if $d$ is even and the $\alpha_{i}$ 's and the $\beta_{j}$ 's are obtained from one another by adding $1 / 2$ modulo $\mathbb{Z}$, then $H(\alpha, \beta \mid 1)$ decomposes as a sum of two motives of specified degrees. In our case, we know a priori that $M=M_{8} \oplus M_{6}$. Factorization (EulerFactor $(L, 3)$ ) then yields $f_{3}(x)$ in two seconds:

$$
\begin{aligned}
(1-268 \cdot 3 x+ & 204193 \cdot 3^{4} x^{2}-1001800 \cdot 3^{9} x^{3}+204193 \cdot 3^{19} x^{4} \\
& \left.-268 \cdot 3^{31} x^{5}+3^{45} x^{6}\right) \\
(1+2992 \cdot x+ & 39116 \cdot 3^{4} x^{2}-7596496 \cdot 3^{6} x^{3}-203836426 \cdot 3^{12} x^{4} \\
& \left.-7596496 \cdot 3^{21} x^{5}+39116 \cdot 3^{34} x^{6}+2992 \cdot 3^{45} x^{7}+3^{60} x^{8}\right)
\end{aligned}
$$

Thus, $M_{6}$ and $M_{8}$ are both irreducible. Moreover Newton-over-Hodge forces the Hodge vector of $M$ to decompose nicely into $h_{6}+h_{8}$ with

$$
\begin{aligned}
& h_{6}:=(0,1,0,1,0,1,0,0,0,0,1,0,1,0,1,0), \\
& h_{8}:=(1,0,1,0,1,0,1,0,0,1,0,1,0,1,0,1) .
\end{aligned}
$$

Likewise, but in 30 seconds, 8 minutes, and 2.5 hours now,

$$
\begin{aligned}
f_{5}(x) & =\left(1+1614 \cdot 5^{3} x+\cdots+5^{45} x^{6}\right)\left(1-41208 x+\cdots+5^{60} x^{8}\right) \\
f_{7}(x) & =\left(1+248232 \cdot 7 x+\cdots+7^{45} x^{6}\right)\left(1+667104 x+\cdots+7^{60} x^{8}\right) \\
f_{11}(x) & =\left(1-883812 \cdot 11 x+\cdots+11^{45} x^{6}\right)\left(1+34438544 x+\cdots+11^{60} x^{8}\right) .
\end{aligned}
$$

Any two of the $f_{p}(x)$ are completely different Galois-theoretically, implying that the two factor $M_{k}$ each have motivic Galois group as large as possible, namely $G S p_{k}$. 
$L$ has a functional equation with respect to $s \leftrightarrow 16-s$. Sign (L) immediately returns 1 , so the analytic rank $r$ of $L$ is even. Evaluate $(\mathrm{L}, 8)$ takes four seconds and returns 0.000000000 , so $r \geq 2$. Evaluate $(\mathrm{L}, 8:$ Derivative: $=2)$ takes fourteen seconds and returns 7.851654518, so $r=2$. The Hardy $Z$-function $Z(t)$ is a vertically rescaled version of $L(M, 8+t i)$. On $[0,7]$ it graphs out to

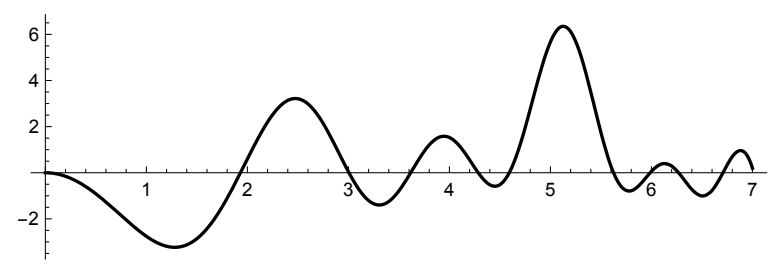

The double zero at $t=0$ is visible. The next three zeros are $\gamma_{1} \approx 1.93195000805$, $\gamma_{2} \approx 3.00559765$, and $\gamma_{3} \approx 3.61679$. Note that this calculation does not give any hints as to the desired factorization $Z(t)=Z_{6}(t) Z_{8}(t)$. In other words, we do not know which motive a given $\gamma_{i}$ belongs to.

\section{The explicit formula}

Let $M$ be a motive of odd weight $w$ with $L$-function assumed to satisfy the Riemann hypothesis. Then its Hodge vector $h$, conductor $N$, analytic rank $r$, Frobenius traces $c_{p^{e}}=\operatorname{Tr}\left(\operatorname{Fr}_{p}^{e} \mid M\right)$, and zeros $1 / 2+\gamma_{k} i$ in the upper half plane are related by $\log N=$

$$
2 \pi r \widehat{F}(0)+4 \pi \sum_{k} \widehat{F}\left(\gamma_{k}\right)+4 \sum_{j>0} h^{j} \int_{0}^{\infty} \widehat{F}(t) E_{j}(t) d t+2 \sum_{p^{e}} c_{p^{e}} \frac{\log p}{p^{(e w+e) / 2}} F(e \log p) .
$$

Here $F$ is an allowed test function, $E_{j}(t)=\log 2 \pi-\Psi((1+j) / 2+i t)$ with $\Psi(s)=$ $\operatorname{Re}\left(\Gamma^{\prime}(s) / \Gamma(s)\right)$, and $h^{p-q}=h^{p, q}$.

The standard Odlyzko test function and its Fourier transform are

$$
F_{\mathrm{Od}}(x)=\chi_{[-1,1]}\left((1-|x|) \cos (\pi x)+\frac{\sin |\pi x|}{\pi}\right), \quad \widehat{F}_{\mathrm{Od}}(t)=\frac{4 \pi \cos ^{2}(t / 2)}{\left(\pi^{2}-t^{2}\right)^{2}} .
$$

Also allowed are the scaled functions $F_{z}(x)=F_{\mathrm{Od}}(x / \log z)$ and their Fourier transforms $\widehat{F}_{z}(t)=(\log z) \widehat{F}_{\mathrm{Od}}(t \log z)$.

\section{Applying the explicit formula to $M_{6}$ and $M_{8}$}

Computing $c_{p^{e}}$ for our motive $M$ is easily done by Magma. However, to get the decomposition $c_{p^{e}}=c_{p^{e}}^{6}+c_{p^{e}}^{8}$, even for just $e=1$, we need to factor $f_{p}(x)$, which we can do only for $p \leq 11$. From the factorizations above, one has $c_{3}^{6}=268 \cdot 3$, 
$c_{9}^{6}=(268 \cdot 3)^{2}-2\left(204193 \cdot 3^{4}\right)$, etc. The explicit formula using $\left(F_{13}, \hat{F}_{13}\right)$, with all terms divided by $\log 2$ for greater clarity, answers Questions 1 and 2:

\begin{tabular}{c|rr|rr|l} 
& term $_{6}$ & $\begin{array}{r}\text { (Tends to } \\
\text { 6 or 7) } \\
\text { total }_{6}\end{array}$ & \multicolumn{1}{|r}{$\begin{array}{r}\text { (Tends to } \\
\text { 8 or 9) } \\
\text { total }\end{array}$} & Comments \\
\hline$h$ & 3.11142 & 3.11142 & 4.85928 & 4.85928 & Hodge contribution \\
\hline 3 & 0.17011 & 3.28154 & -0.63306 & 4.22622 & Contributions \\
5 & -0.35472 & 2.92682 & 0.07245 & 4.29897 & from the successively \\
7 & -0.07386 & 2.85296 & -0.02836 & 4.27031 & harder factorizations \\
9 & -0.02269 & 2.83027 & 0.00183 & 4.27214 & of Frobenius \\
11 & 0.00028 & 2.83055 & -0.00101 & 4.27114 & polynomials $f_{p}(x)$ \\
\hline \hline$r$ & 2.99946 & 5.83002 & 2.99946 & 7.27060 & Forced! A1: $(1,1)$ \\
\hline$\gamma_{1}$ & & 5.83002 & 1.68061 & 8.95121 & Forced! A2: $\left(2^{6}, 2^{9}\right)$ \\
$\gamma_{2}$ & 0.13610 & 5.96612 & & 8.95121 & Forced! \\
$\vdots$ & $\vdots$ & $\vdots$ & $\vdots$ & $\vdots$ & \\
Total & & 6.00000 & & 9.00000 &
\end{tabular}

Terms are positive after the double line, and so these terms must be associated with either $M_{6}$ or $M_{8}$ so as to keep (total 6, total $_{8}$ ) coordinatewise less than either $(6,9)$ or $(7,8)$. This forces the indicated answers. Thus, both motives have analytic rank 1 . The prime 2 is tamely ramified in $M_{6}$ and minimally wildly ramified in $M_{8}$

Remarkably, the talk just described relates directly to two collaborative projects begun at the Matrix Institute. The decomposition studied here is the $d=16$ case of the sequence of decompositions mentioned in $\S 4$.ii in the abstract with Rodriguez Villegas. The Hodge vectors $h_{6}$ and $h_{8}$ also arise for the $L$-functions denoted $L_{16}$ and $L_{18}$ in the abstract with Broadhurst; conductors there are $1260=2^{2} \cdot 3^{2} \cdot 5 \cdot 7$ and $7560=2^{3} \cdot 3^{3} \cdot 5 \cdot 7$ respectively.

\section{Acknowledgements}

I thank the organizers and local staff for the excellent conference. My attendance at the Matrix Institute was supported by the conference and by grant DMS-1601350 from the National Science Foundation.

\section{References}

1. J.-F.-Mestre. Formules explicites et minorations de conducteurs de de variétés algébriques, Compositio Mathematica, 58 (1986) 209-232.

2. W. Bosma, J. J. Cannon, C. Fieker, A. Steel (eds.) Handbook of Magma functions, Edition 2.19 (2013), 5291 pages. 